\title{
AVALIAÇÃO DO IMPACTO AMBIENTAL CAUSADO PELA PRODUÇÃO DE AÇO USANDO O MÉTODO RECIPE
}

\author{
Lincoln Martins Godoy ${ }^{1}$ \\ José Tomadon Júnior ${ }^{2}$
}

Cássia Maria Lie Ugaya ${ }^{3}$

\begin{abstract}
RESUMO
A Avaliação do Ciclo de Vida (ACV) avalia os aspectos e os potenciais impactos ambientais ao longo do ciclo de vida de um produto. Esta avaliação é realizada desde a extração da matéria-prima da natureza, passando pela produção, uso, tratamento de fim de vida, reciclagem e disposição final. Para realizar a ACV, métodos de quantificação do potencial impacto ambiental podem ser utilizados, destaca-se: EDIP 97/2003; IMPACT 2002+; CML-IA; ReCiPe; entre outros. Deste modo, este artigo tem o objetivo de avaliar o impacto ambiental causado pela produção de aço utilizando o software SimaPro. O processo de produção do aço é modelado no software SimaPro e o método utilizado para calcular o potencial impacto ambiental foi o ReCiPe. Na base de dados do software (base Ecolnvent v.3) obtevese os dados referentes as entradas e saídas do processo de produção. Os resultados mostram que o processo de produção do aço causa danos maiores na categoria de impacto referente a danos aos recursos, em especial a depleção de metais e recursos fósseis, sendo que o níquel é a substância que mais contribui para a depleção de recursos.
\end{abstract}

PALAVRAS-CHAVE: Avaliação do Ciclo de Vida. Aço. ReCiPe. SimaPro.

\section{ASSESSMENT OF ENVIRONMENTAL IMPACT CAUSED BY THE STEEL PRODUCTION USING RECIPE METHOD}

\footnotetext{
${ }_{1}^{1}$ Engenharia Mecânica, Universidade Tecnológica Federal do Paraná. E-mail:lincoln.godoy@gmail.com ${ }^{2}$ Formação acadêmica, Universidade Tecnológica Federal do Paraná. E-mail: jtjunior@utfpr.edu.br

${ }^{3}$ Formação acadêmica, Universidade Tecnológica Federal do Paraná. E-mail: cassiaugaya@utfpr.edu.br
} 


\begin{abstract}
Life cycle assessment (LCA) estimates the aspects and potential environmental impacts throughout a product's life cycle. This assessment goes from raw material extraction, through production, use, end-oflife treatment, recycling and final disposal. To implement $L C A$, quantification methods of potential environmental impact can be used, among them are EDIP 97/2003; IMPACT 2002+; CML-IA; ReCiPe; among others. Thus, this article has the goal to assess the environmental impact caused by the production of steel using SimaPro software. The process of steel production is modeled at the software SimaPro and the method used to calculate the potential environmental impact was ReCiPe method. Through the software database (base Ecoinvent v.3) data are found in relation to the multiples inputs and outputs of the production process. The results shows that the steel production process yields bigger damages on the category related to the damages to resource availability, in particular, metal and fossil depletion, having nickel as the substance that contributes the most to the resources depletion.
\end{abstract}

KEY-WORDS: Life Cycle Assessment. Steel. ReCiPe. SimaPro.

\title{
EVALUACIÓN DE IMPACTO AMBIENTAL PROVOCADA POR LA PRODUCCIÓN DE ACERO DE UTILIZAR LA RECETA MÉTODO
}

\section{RESUMEN}

El Análisis de Ciclo de Vida (ACV) evalúa los aspectos e impactos ambientales potenciales en todo el ciclo de vida de un producto. Esta revisión se lleva a cabo desde la extracción de materias primas de la naturaleza, a través de la producción, uso final del tratamiento vida, reciclaje y disposición final. Para realizar la LCA, los métodos de cuantificación del impacto ambiental potenciales se pueden utilizar, se destaca: Edip 97/2003; IMPACTO 2002+; CML-IA; ReCiPe, entre otros. Por lo tanto, este artículo tiene como objetivo evaluar el impacto ambiental de la producción de acero mediante el software SimaPro. El proceso de producción de acero sigue el modelo del software SimaPro y el método utilizado para calcular el potencial impacto ambiental era la receta. En la base de datos de software (base ecoinvent v.3) dio los datos de entrada y salidas del proceso de producción. Los resultados muestran que el proceso de producción de acero provoca un mayor daño a la categoría de daños por impacto relacionado con los recursos, en particular, el agotamiento de los recursos fósiles y metales, y el níquel es la sustancia que contribuye al agotamiento de los recursos.

PALABRAS CLAVE: Evaluación del Ciclo de Vida. Acero. ReCiPe. SimaPro.

\section{INTRODUÇÃO}

De acordo com Ferreira (2004), o termo Avaliação do Ciclo de Vida foi empregado pela primeira vez na década de 90 nos Estados Unidos da América. Porém um dos primeiros estudos que quantificavam os recursos necessários, suas 
emissões e os resíduos ocasionados foi coordenado pelo Midwest Reseach Institute (MRI) em 1969. Na década de 90 surgem os primeiros movimentos para a normalização da ACV. Em 1997 surge a primeira norma da Organização Internacional para Padronização (ISO), a ISO 14040. No Brasil, a tradução desta norma só chegou em 2001 (ABCV, 2014).

Avaliação do Ciclo de Vida (ACV) é uma técnica que avalia os aspectos e os potenciais impactos ambientais ao longo do ciclo de vida de um produto, desde a aquisição de matéria-prima, passando pela produção, uso, tratamento de fim de vida, reciclagem e disposição final. Geralmente, a ACV não aborda os aspectos econômicos ou sociais de um produto e pode não ser a técnica mais adequada a ser utilizada em determinadas situações (ISO, 2006).

Segundo Marzullo (2007), não há um método único de condução dos estudos da ACV. Por este motivo, as normas da série ISO 14040 sugerem o enfoque nas quatro principais etapas da metodologia: definição de objetivo e escopo, análise do inventário, avaliação de impactos e interpretação.

A ACV oferece uma oportunidade de vislumbrar os potenciais impactos ambientais de uma cadeia produtiva. A cadeia produtiva do aço pode então ser avaliada utilizando esta técnica visto que as etapas do processo redução em altoforno, sinterização e oxidação na aciaria geram grande emissão de poluentes para a atmosfera.

Ugaya (2001), cita que o processo de produção do aço "consiste basicamente do beneficiamento, da aglomeração por pelotização e sinterização, da redução e da oxidação".

A redução em alto-forno é a primeira etapa para a produção do aço. Esta fase gera como produto uma liga ferro-carbono, denominada ferro gusa. Esta liga, ainda em estado líquido, é conduzida à aciaria, onde em fornos apropriados, é transformado em aço (CHIAVERINI, 1986). Segundo Milanez (2008) os principais impactos causados pela etapa do alto-forno ocorrem devido à necessidade de carvão vegetal para a execução do processo. 
Durante o processo de queima do carvão, dióxido de carbono e metano são liberados, contribuindo para o aumento da quantidade de carbono na atmosfera. Ainda está presente a emissão de óxidos de enxofre e óxidos de nitrogênio que, combinados com a umidade do ar formam ácidos de enxofre e ácidos de nitrogênio, fenômeno conhecido como "chuva ácida". (MILANEZ, 2008).

Estas particularidades do processo justificam o objetivo deste estudo: Avaliar o processo de produção de aço utilizando o software SimaPro com o emprego do método ReCiPe para o cálculo do potencial impacto ambiental.

\section{MATERIAIS E MÉTODOS}

Esta pesquisa é classificada quanto aos fins como descritiva e explicativa e, quanto aos meios, como bibliográfica. Foram realizados estudos sobre o processo de produção do aço e sobre a Avaliação do Ciclo de Vida do mesmo.

Os dados necessários para a avaliação do processo de produção do aço foram obtidos na base de dados Ecoinvent 3, implementada no software SimaPro versão 8, licença Faculty. Para os cálculos, foi utilizado o método ReCiPe versão 1.1 individualista, fazendo uso de normalização ${ }^{4}$ e ponderação ${ }^{5}$ global, o mais indicado para retratar a realidade brasileira. Este método possui dois conjuntos de categorias de impacto de acordo com a sua abordagem: ponto médio (mid-point) e ponto final (end-point). Neste artigo, discute-se apenas os impactos em ponto final.

A abordagem ponto final baseia-se na identificação da gravidade ou das consequências das categorias de impacto de ponto médio nas áreas de proteção a nível de ponto final. Estas áreas de proteção se referem a: Dano a Saúde Humana; Dano ao Ecossistema; e Danos aos Recursos. Como os métodos de ponto final são

\footnotetext{
${ }^{4}$ Cálculo da magnitude dos resultados dos indicadores de categoria com relação a informações de referência (ISO, 2006).

${ }^{5}$ Conversão e possível agregação dos resultados dos indicadores entre as diferentes categorias de impacto utilizando fatores numéricos baseados em escolha de valores; convém que os dados anteriores à ponderação permaneçam disponíveis (ISO, 2006).
} 
diretamente relacionados ao dano, estes possuem maior importância na tomada de decisão, porém possuem alta subjetividade (PIEKARSKI et al, 2012).

Neste estudo, analisou-se os impactos relacionados à produção de $1 \mathrm{~kg}$ de aço cromo 18/8 (aço com $18 \%$ de cromo e $8 \%$ de níquel em sua composição). $\mathrm{Na}$ etapa de avaliação de danos, para que seja possível identificar quais substâncias contribuem majoritariamente para o impacto, usou-se uma fração de corte de $0,1 \%$ do processo.

\section{RESULTADOS E DISCUSSÃO}

Utilizando a base de dados Ecoinvent 3, presente no software SimaPro, obteve-se primeiramente os resultados do potencial impacto ambiental da produção do aço para as categorias de Dano a Saúde Humana, Qualidade do Ecossistema e Danos aos Recursos.

A Figura 1 apresenta o potencial impacto ambiental na categoria de Dano a Saúde Humana. O valor total do potencial impacto nesta categoria foi de 1,31E-05 DALY, que significa anos perdidos por incapacidade. O método ReCiPe considera que o Dano a Saúde Humana é causado pelas seguintes categorias de ponto médio: Mudanças Climáticas (Saúde Humana), Depleção de Ozônio, Toxicidade Humana, Formação de Oxidantes Fotoquímicos, Formação de Particulados e Radiação Ionizante (Figura 1). 


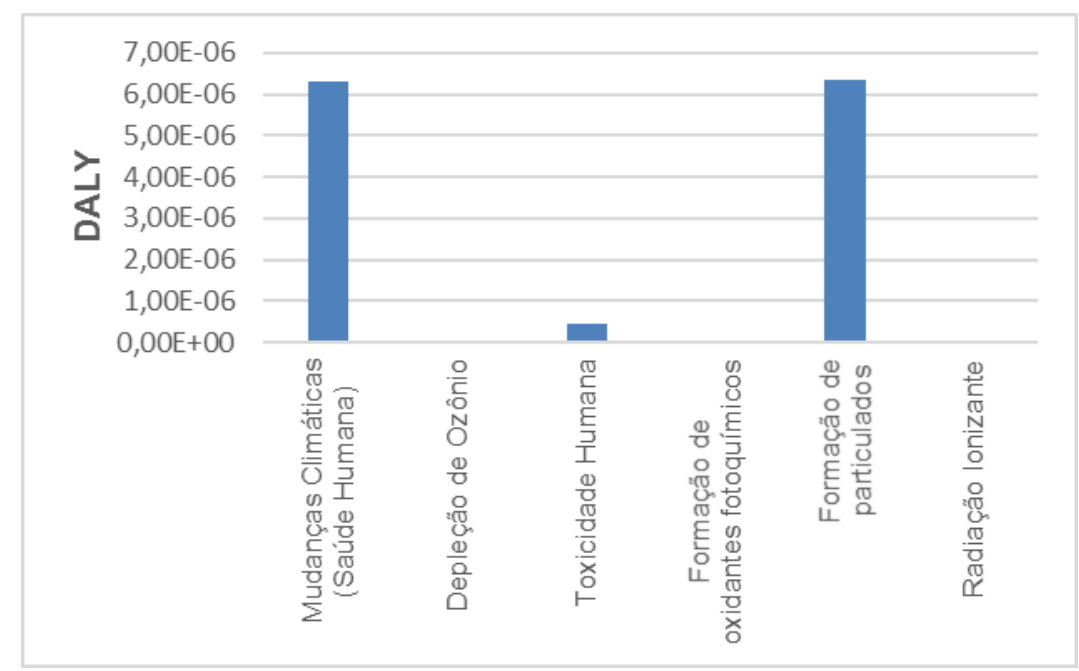

Figura 1. Impacto ambiental causado pela produção de aço na categoria de Dano a Saúde Humana.

As categorias que apresentam maior impacto a Saúde Humana são Mudanças Climáticas e Formação de Particulados, que apresentaram um impacto de 6,32E-06 DALY e 6,36E-06 DALY respectivamente.

O Dano ao Ecossistema é dividido entre as categorias Mudanças Climáticas (Ecossistema), Acidificação Terrestre, Eutrofização de Água Doce, Ecotoxicidade Terrestre, Ecotoxicidade de Água Doce, Ecotoxicidade Marinha, Ocupação de Terras Agrícolas, Ocupação de Terras Urbanas e Transformação Natural da Terra. O potencial impacto ambiental total nesta categoria foi de 4,64E-08 espécies/ano. A Figura 2 apresenta os valores correspondentes a cada uma das categorias. 


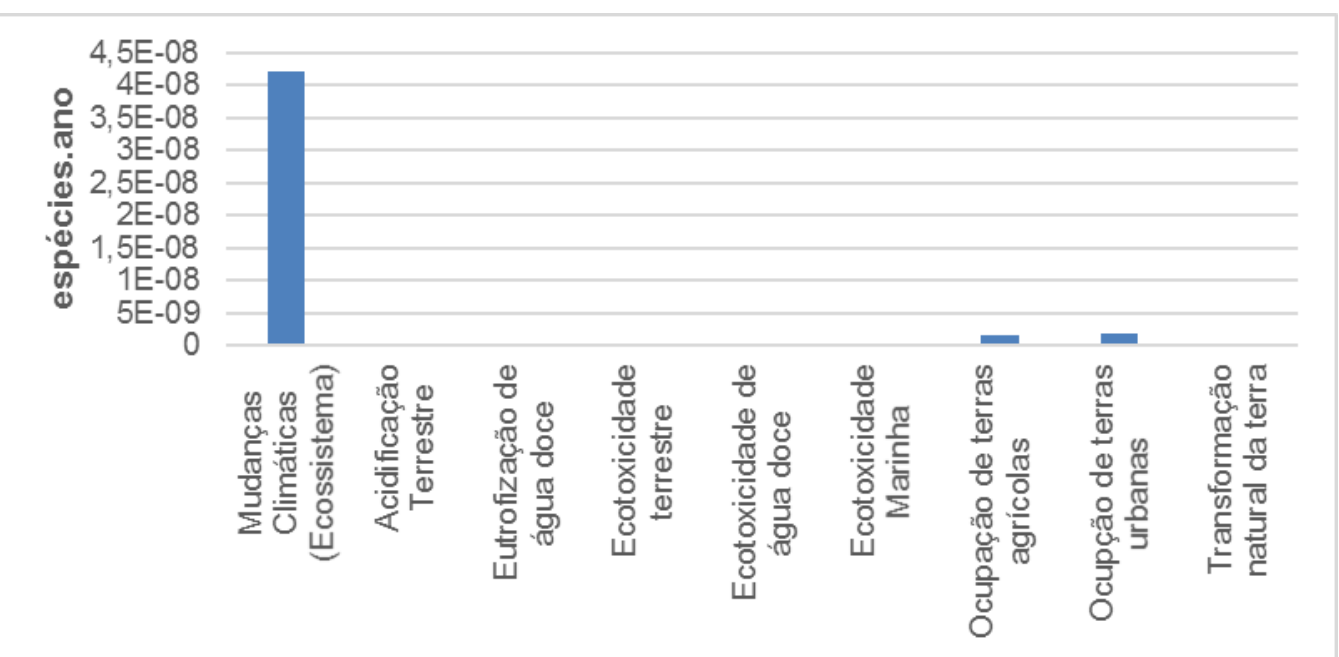

Figura 2. Impacto ambiental causado pela produção de aço na categoria de Qualidade do Ecossistema.

As categorias que apresentaram maior impacto a qualidade do ecossistema foram: Mudanças Climáticas, com um impacto de 4,21E-08 espécies/ano; Ocupação de Terras Urbanas, com 1,76E-09 espécies/ano; e Ocupação de Terras Agrícolas, que apresentou um impacto de 1,59E-09 espécies/ano.

O potencial impacto ambiental total na categoria de dano aos Recursos foi de $\$ 1,032623$, sendo dividido entre as categorias Depleção de metal e Depleção fóssil, como apresentado na Figura 3.

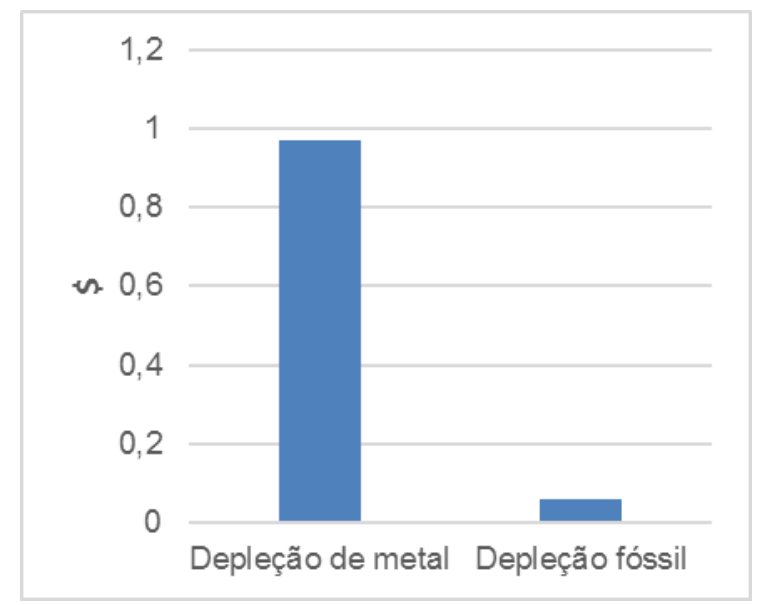

Figura 3. Impacto ambiental causado pela produção de aço na categoria de dano aos Recursos. 
Dilemas da Sustentabilidade Urbana

A categoria referente a Depleção de metal foi a que apresentou maior impacto ambiental aos recursos, com o total de $\$ 0,97258$.

Nota-se que cada grupo de dados referentes à cada uma das três categorias de danos tem sua própria unidade de medida. Para fins de comparação e identificação de qual categoria teria maior potencial impacto ambiental, os resultados foram normalizados. Os resultados da normalização estão apresentados na Figura 4.

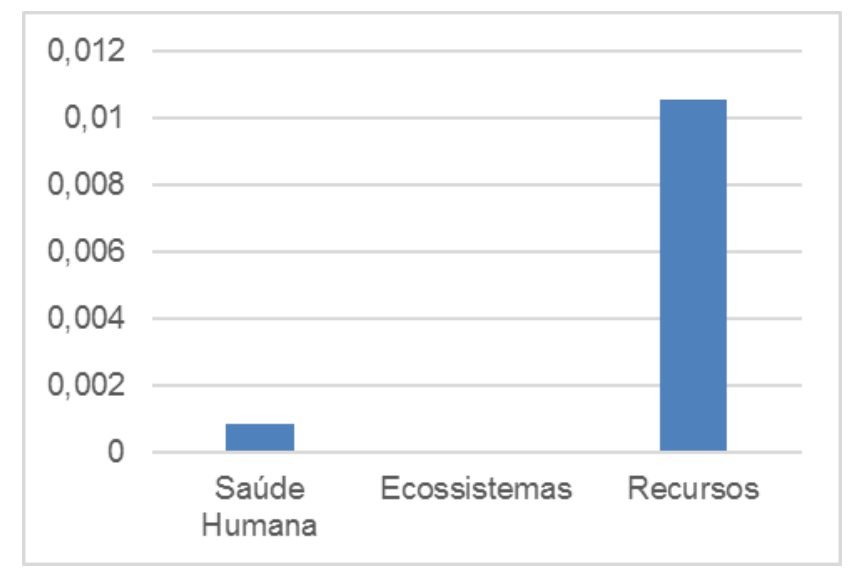

Figura 4. Potencial impacto ambiental da produção do aço nas categorias de dano normalizado.

Graficamente, fica claro que a categoria que mais impacta o ambiente é a referente aos Recursos, com um valor de 0,01053. Através do software é possível obter mais informações desta categoria, conhecer quais substâncias que mais contribuem para a depleção dos recursos. Para isso, empregou-se uma fração de corte de $0,1 \%$ do processo, o que facilita a obtenção das substâncias que contribuem de forma majoritária ao impacto. Estas substâncias são mostradas na Tabela 1. 
Tabela 1.Principais substâncias contribuintes para a depleção de recursos.

\begin{tabular}{c|c|c} 
Substâncias & Unidade & Total \\
\hline $\begin{array}{c}\text { Níquel, } 1.98 \% \text { em } \\
\text { silicatos, } 1.04 \% \text { em } \\
\text { minério bruto }\end{array}$ & $\$$ & 0,498889379 \\
\hline Cromo & $\$$ & 0,43459177 \\
\hline Ferro & $\$$ & 0,028870761 \\
\hline Carvão, duro & $\$$ & 0,028290688 \\
\hline Gás, natural/m3 & $\$$ & 0,016763764 \\
\hline Petróleo, bruto & $\$$ & 0,012947422 \\
\hline $\begin{array}{c}\text { Cobre, 0.99\% em sulfeto, } \\
\text { Cu 0.36\% e Mo 8.2E-3\% } \\
\text { em minério bruto }\end{array}$ & $\$$ & 0,004190668 \\
\hline Carvão, marrom & $\$$ & 0,001525768 \\
\hline Manganês & $\$$ & 0,001297962 \\
\hline
\end{tabular}

Entre as substâncias apresentadas na Tabela 1, o níquel, o cromo e o ferro são as que mais contribuem para a depleção dos recursos, sendo o níquel o maior contribuinte, com um valor total de $\$ 0,498889379$, seguido pelo cromo e o ferro, cada um com o valor de $\$ 0,43459177$ e $\$ 0,028870761$ respectivamente.

\section{CONCLUSÃO}

O objeto de estudo deste artigo foi a produção de aço cromo $18 / 8$ e, com base na avaliação de inventário e nos resultados da avaliação de impacto podemos concluir que o processo de produção de $1 \mathrm{~kg}$ do aço gera danos consideráveis ao ambiente.

Analisando os dados obtidos através do software SimaPro, pelo método ReCiPe, observa-se que as categorias de impacto cujos danos foram mais significativos ao ambiente foram as Mudanças Climáticas (Saúde Humana), Formação de particulados, Mudanças climáticas (Ecossistemas) e Depleção de metal (Recursos).

Utilizando dados normalizados, notou-se que entre as três categorias de danos abrangidas pelo método $\mathrm{ReCiPe}$, a categoria referente à depleção de recursos é a que mais gera potencial impacto ambiental. Nesta categoria estão relacionadas as 
categorias de impacto que tratam da depleção de metais e recursos fósseis, sendo que as principais substâncias consumidas foram o níquel, o cromo e o ferro.

\section{REFERÊNCIAS}

ASSOCIAÇÃO BRASILEIRA DE CICLO DE VIDA. Histórico do desenvolvimento da ACV. Disponível em:<http://abcvbrasil.org.br/historia-acv.php> Acesso em: 09 jun. 2014.

CHIAVERINI, Vicente. Tecnologia mecânica. 2.ed. São Paulo: McGraw-Hill, 1986. v. 3.

FERREIRA, José V. F. Análise de ciclo de vida dos produtos. Instituto Politécnico de Viseu, 2004.

INTERNATIONAL ORGANIZATION FOR STANDARDZATION. ISO 14040: Environmental management - Life cycle assessment - Principles and framework. Suíça, 2006.

INTERNATIONAL ORGANIZATION FOR STANDARDZATION. ISO 14044: Environmental management — Life cycle assessment — Requirements and guidelines. Suíça, 2006.

MARZULLO, Rita de C. M. Análise de ecoeficiência dos óleos vegetais oriundos da soja e palma, visando a produção de biodiesel. 2007. 302 f. Dissertação (Mestrado em Engenharia) - Universidade de São Paulo, Escola Politécnica, São Paulo, 2007.

MILANEZ, Bruno. Siderurgia, sociedade e meio ambiente. ECOA - Ecologia e Ação. Disponível em: <http://www.riosvivos.org.br/Noticia/Siderurgia++sociedade+e+meio+ambiente/12591> Acesso em: 29 mai. 2014.

PIEKARSKI, Cassiano M. et al. Métodos de avaliação de impactos do ciclo de vida: uma discussão para adoção de métodos nas especificidades brasileiras. Revista Gestão Industrial. Ponta Grossa, 2012.

UGAYA, Cássia M. L. Análise de ciclo de vida: estudo de caso para materiais e componentes automotivos no Brasil. 2001. 199 f. Tese (Doutorado em Engenharia Mecânica) - Faculdade de Engenharia Mecânica, Universidade Estadual de Campinas, Campinas, 2001. 